\title{
A Holographic-based Model for Logistics Resources Integration
}

\author{
Daqing GONG, Shifeng LIU \\ School of Economics \& Management, \\ Beijing Jiaotong University, China, \\ gongtuipigua@163.com; shfliu@bjtu.edu.cn
}

\begin{abstract}
This study investigates the logistics resource integration problem. Based on a comprehensive literature review, we find that there is much room for improvement regarding the robustness problems in logistics resources integration. Logistics resources integration should especially consider uncertainties. In this study, we propose a holographic-based model (Internet of Things and Neural Network) to illustrate the problem. Internet of Things (IoT) is able to receive real-time data (including uncertainty information) in logistics systems and is equivalent to the perception subsystem. Neural Network, on the other hand, can determine the overall operation state for logistics resources integration and plays the role of analysis and assessment. Through simulation, this study shows that real-time data in logistics systems are transmitted based on protocols, so that uncertainty information can be received by the IoT model. The Neural Network model can comprehensively evaluate uncertainties through the neural network algorithm. Therefore, the robustness of logistics resources integration can be ensured in the logistics system.
\end{abstract}

Keywords: logistics resources integration, holographic, Internet of Things (IoT), Neural Network

\section{Introduction}

Logistics resource usually refers to various logistics infrastructure. However, it also includes an organization's production factors like capital, technologies, knowledge, information, personnel, equipment, customer, organization, culture, etc. in logistics services and working processes. According to $\mathrm{Xu}$ (2005), all of the economic systems, social systems, and natural systems are logistics resources in the view of material flow. With the development of logistics industry, logistics system will become more and more complex. Under these circumstances, the problems of logistics resources integration need to be solved urgently.

Based on the findings from existing literatures, we found that the existing studies discuss logistics system resources in natural languages or focus on the application of mathematics and information technologies in logistics. However, the quantitative description of complex logistics resources cannot be substituted by natural languages, mathematics or information technologies. In this study, we focus on the 'robustness', which refers to the rapid acquisition, learning, feeding back, and intelligent analysis towards a variety of uncertainties information. According to Klibi et al. (2010), the development of a comprehensive SCN (Supply Chain Network) design methodology should consider uncertainty sources and risk exposures. Therefore Manuj and Mentzer (2008) discussed that high-impact extreme events should not be treated in the same way as low-impact business-as-usual events. Lempert et al. (2006) discussed that it is possible to elaborate plausible future scenarios under deep uncertainties. Similarly, Snyder (2006) defined robustness as the extent in which a SCN is able to carry its functions for a variety of plausible future scenarios. The question remains on how we can tackle this problem. Tomlin (2006) suggested flexible sourcing contracts as a hedge against randomness and hazards, in order to increase the SCN expected value. Sheffi (2005) used the physical insurance against SCN's risk exposure to circumvent disruptions as much as possible, as well as to bounce back quickly when struck. So the research questions in this study are: how to realize the quantitative descriptions of the complex logistics resources and how to make the SCN robust. At present, the aforementioned problems have not been resolved yet in related research works.

In this study, we propose a holographic-based model to illustrate the problems of logistics resource integration. We organize this paper as follows: in the following section, we present a comprehensive review, which forms the theoretical foundation of this study. In section 3, we present the analytical models as 
well as the simulation results through which we verify the model. Finally, we conclude the study in section 4 .

\section{Literature Review}

Logistics resources integration aims to integrate different resources in the value chain, which consists of capital chain, physical chain, business chain and operation chain. Findings from existing literature indicate that logistics resources integration mainly includes goals setting, resources recognizing, resources obtaining and resources maintaining.

Literature on goals setting and resources recognizing in the supply chain management perspective proposes that supply chain management should involve material flows, information flows, and finance flows in a network consisting of customers, suppliers, manufacturers, and distributors (Lee, 2000). Furthermore, material flows include both physical product flows from suppliers to customers in the chain and reverse flows via product returns, servicing, recycling, and disposal. Information flows involve order transmission and delivery status. Financial flows include credit terms, payment schedules, etc. So effectively coordinating and integrating this flow is essential for effective supply chain management. Lepak and Smith (2007) discussed the deep-seated meaning of logistics resources integration in terms of the goals of value creation, the process of value creation, and the process of value capture, based on a society, organization, and individual level.

There is more literature on topics around resources obtaining, but most of them are particularly quantitative models neglecting the robustness of logistics system. However, quantitative description of a complex logistics system cannot be substituted by mathematics or others. We can see them as follows:

The multi-agent negotiation model: Brintrup (2010) established the supplier selection model of multi-agent, multi-target, and multirole with the aim to reduce transaction time and to increase corporate revenue. From the perspective of customer needs, Akanle and Zhang (2008) proposed to optimize the configuration for supply chains and they built a multi-agent model coordinating iterative bidding mechanism based on interactive algorithms, and obtained the optimum based on genetic algorithm. On the background of global supply chain manufacture network, Jiao, You and Kumar (2006) set up a multiagent (upstream and downstream enterprises involved in a supply chain), multi-contracting negotiations (customer demand) model, which is helpful for dealing with spontaneous or semi-spontaneous problems in supply chains. So we can conclude that the quantitative model in the above literature is agent and genetic algorithm-based.

The simulation-based model: Ploog and Spengler (2002) assumed the electronic products recovery mode and developed a mathematical model with an application involving lingo procedures. The raw materials management model was built by Tserng, Yin and Li (2006), this model described its objectives and constraints, and subsequently simulations were carried out based on Visual Basic 6.0 and Microsoft SQL, discovering that the structure of supply chain inventory cost is mostly affected by the total inventory cost. Bhattacharyya (2011) also analyzed the supply chain cost problem, and developed the LINGO program for simulation. In the end, he found the optimal integration path. So we can conclude that the quantitative model in the above literature is to obtain solutions for complex mathematical model.

The System Dynamics (SD) model: Dyson and Chang (2005) studied the closed-loop logistics system for solid waste as a SD application. Zanjani, et al. (2012) continued to analyze the waste management based on SD and they demonstrated that public participation was a very effective way for waste management. Based on the idea of closed-loop logistics, the SD model of wasted electronic products recycling was set up by Georgiadis and Besiou (2010) to carry out sensitivity analysis of different factors. Thus $\mathrm{SD}$, as one of the continuous system modeling methods, is applicable to specific issues-oriented modeling.

The comprehensive model: Schieritz and Größler (2003) developed the contrastive analysis of SD-based model and agent-based model. They thought that the structure of supply chains is an 'emerging', and the 
external structure is determined by the interaction among the main bodies of the supply chain. However, the internal structure is constrained by the conducts rules and environment. Finally, a macroscopic layer of supply chain was built based on agent and SD. A systematic explanation of bulk recycling, disassembly recycling, smelter model recycling is introduced by Sodhi and Reimer (2001). They set benefits as the objective of the model for the different recycling models. The authors also depicted resources, recyclers, processors and demand market of the electronic product recycling system. Subsequently followed by a mathematical model to describe the main bodies' behaviors of the recovery process and lastly they demonstrated the model through examples. Actually, the comprehensive theory is an important method in dealing with resources integration.

Many scholars continued to analyze problems in the view of logistics resources maintaining, and the analysis can be summarized in two aspects: Hard way, which is to build the information network platform by achieving synergies; soft way, which is to create internal incentives by maintaining a fair distribution of benefits. Although the literature has paid attention to the robustness of logistics system, they have not tried to solve it yet. We can see them as follows:

For the hard way: Prajogo and Olhager (2011) proposed the logistics resources integration concept on the background of information. The integration of logistics information resources can drive the integration of logistics resources, information, and information sharing. This integration has a significant impact on supply chain performance. Barton and Thomas (2009) pointed out that supply chain management has become an increasingly important tool to improve the situation of the manufacturing industry. Rapid reaction abilities, intelligent integration system, and management capabilities are all keys to create a robust supply chain. Lee (2000) emphasized on the importance of information resources integration and its contents include: demand, inventory status, production plan, production time, promotion plan, demand forecast, and transportation routes sharing. Wamba and Chatfield (2009) discussed the application of RF technology in the logistics resources integration. So we can conclude that the quantitative model in the above literature is information technology-based.

For the soft way: Handfield and Nichols (1999) and Sahay (2003) considered the trust mechanism is of great importance to supply chain management. Assuming that robust supply chains are needed to ensure mutual trust and responsibilities among the system main bodies for a long time. Furthermore, the sharing of information, risk, and revenue is a must. With the help of cooperative game theory, Nagarajan and Sos 'ic' (2008) analyzed the integration problems of supply chains and proposed the theory of 'vision', which provides a new way for supply chain management. So we can conclude that the quantitative model in the above literatures is game theory-based.

To sum up, existing literatures mainly focus on depicting complex logistics system resources, setting benefits as the ultimate goal for logistics resources integration, or applying information systems to logistics resources integration. Further study on 'robustness' of complex logistics system is necessary to provide insights for practitioners and academics.

\section{Holographic-based Logistics Resources Integration Modeling: Internet of Things and Neural Network}

\subsection{The skeleton of the model}

The quantitative description of complex logistics system resources cannot be substituted by natural languages, mathematical formulas, or information technology. The structural description of complex logistics system has four elements: system nodes, system structure, system communication, and learning/feeding back. To a certain extent, the model is agent-based including Artificial Intelligence and Distributed Systems (Dzitac, I., 2009).

To sum up, the model includes Internet of Things (IoT) and Neural Network. The former is able to receive real-time data in the logistics system and is equivalent to a perception subsystem. The latter determines 
the overall operation state of logistics resources integration and plays the role of analysis and assessment. For more details see Figure 1.

Holographic-based Modeling is characterized by the application of sensing technologies compared with traditional internet and mobile communications networks. Each sensor is a source of information in the network. The information is also served to the neural network and transmitted to the client. For more details see Table 1 .

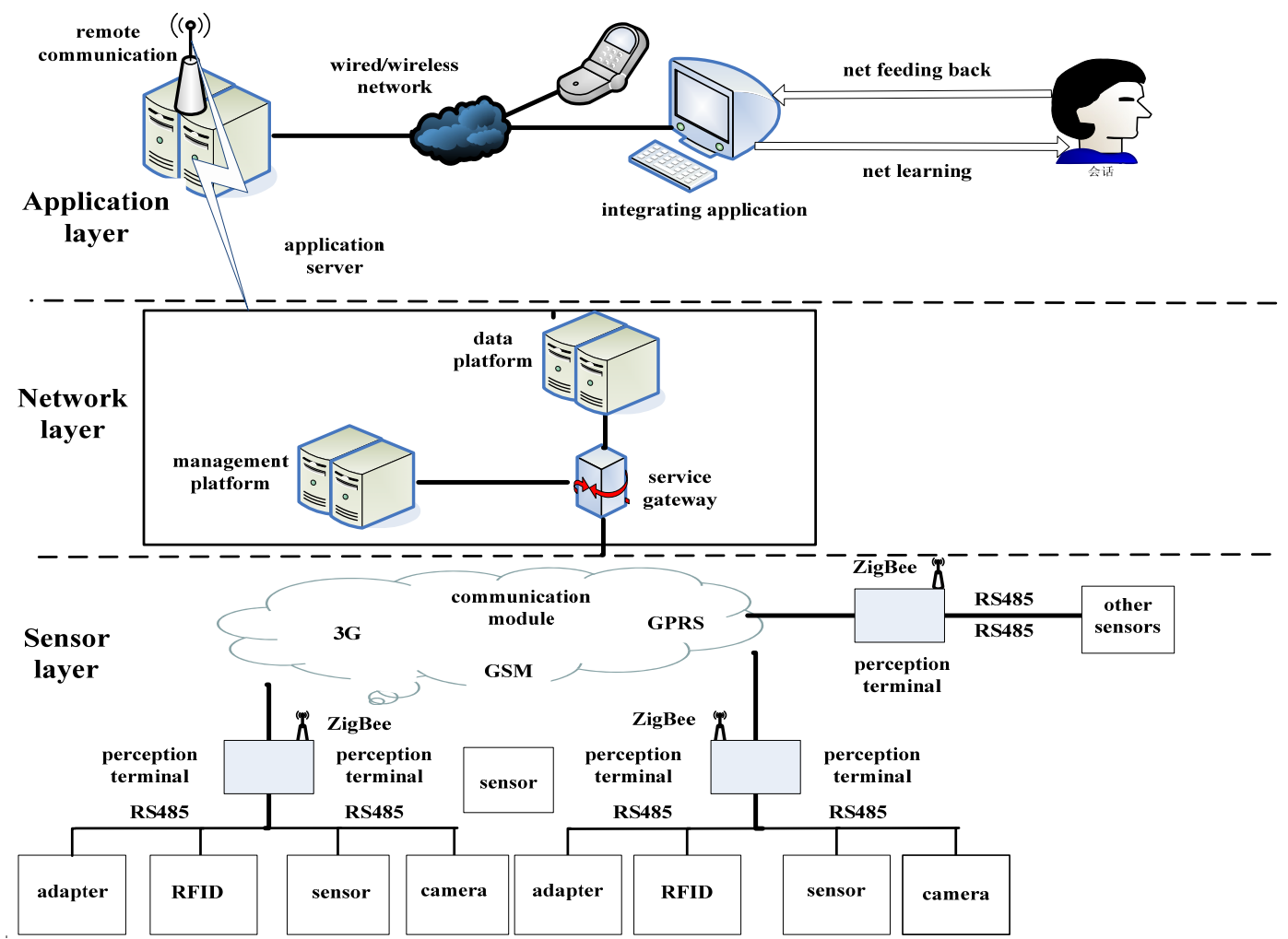

Figure 1. Holographic-based Logistics Resources Integration Model

Table 1. Model Description

\begin{tabular}{|l|l|}
\hline \multicolumn{1}{|c|}{ Model component } & \multicolumn{1}{|c|}{ Descriptions } \\
\hline Adapter & $\begin{array}{l}\text { Analog measurement, Non-numeric information collection and } \\
\text { transformation. }\end{array}$ \\
\hline Sensor & $\begin{array}{l}\text { The device, converting the measured information to the available } \\
\text { output signal in accordance to certain rules. It is usually } \\
\text { comprised by the sensitive elements and transition elements. }\end{array}$ \\
\hline Perception terminal & $\begin{array}{l}\text { To complete the link-up of physics and data for the sensor } \\
\text { module and communication module, pre-treat sensor data, and } \\
\text { transform communication protocols. }\end{array}$ \\
\hline RS485 & $\begin{array}{l}\text { Half-Duplex Communication, Serial Data Interface, Multi-Point } \\
\text { Communication. }\end{array}$ \\
\hline ZIGBEE & $\begin{array}{l}\text { Its floor layer technology cites IEEE 802.15 directly; it is } \\
\text { characterized by low-cost, low-power, short-range, low data rate, } \\
\text { high reliability. }\end{array}$ \\
\hline Communication module & $\begin{array}{l}\text { Communication module contains of two sections. One is the } \\
\text { communication module of extensive sensor layer, such as } \\
\text { ZigBee. Another is the communication module of traditional } \\
\text { communication networks, such as 3G/GSM/GPRS. }\end{array}$ \\
\hline Management platform & $\begin{array}{l}\text { Responsible for the daily maintenance and management of the } \\
\text { sensor layer. }\end{array}$ \\
\hline Data platform & Collects all the data that the sensor layer reports. \\
\hline Application server & Served for logistics resource information sharing. \\
\hline Network learning and feeding back & $\begin{array}{l}\text { Artificial Neural Networks (abbrev. ANNs), is an imitation of } \\
\text { animal neural network behavior. A mathematical model for } \\
\text { distributed and parallel information processing. }\end{array}$ \\
\hline & \\
\hline
\end{tabular}


Therefore, the structural description of the model not only includes the property description, but also includes information collection and system communication. Furthermore, the model can make intelligent analysis and realize feedback towards different kinds of information. Intelligent analysis, on the other hand, refers to that the model can make right judgments under irregular information change.

\subsection{Communication protocol-based complex logistics system data acquisition and transmission}

Data acquisition and transmission are realized through the communication protocol, which consists of SAP (Sensor Application Protocol) and SCP (Sensor Communications Protocol). For more details please see Figure 2.

\subsubsection{Summary of SAP}

The communication between the perception terminal and the platform is realized by the protocol (Figure 3). In the protocol, English characters are coded in ASCII and Chinese characters are coded in GB2312 (the same with $\mathrm{SCP})$. The protocol location of the system is shown in Figure 2.

Thus information can be transmitted by the frame structure as depicted in Figure 3.

The meanings and provisions of the frame structure are shown in Table 2.

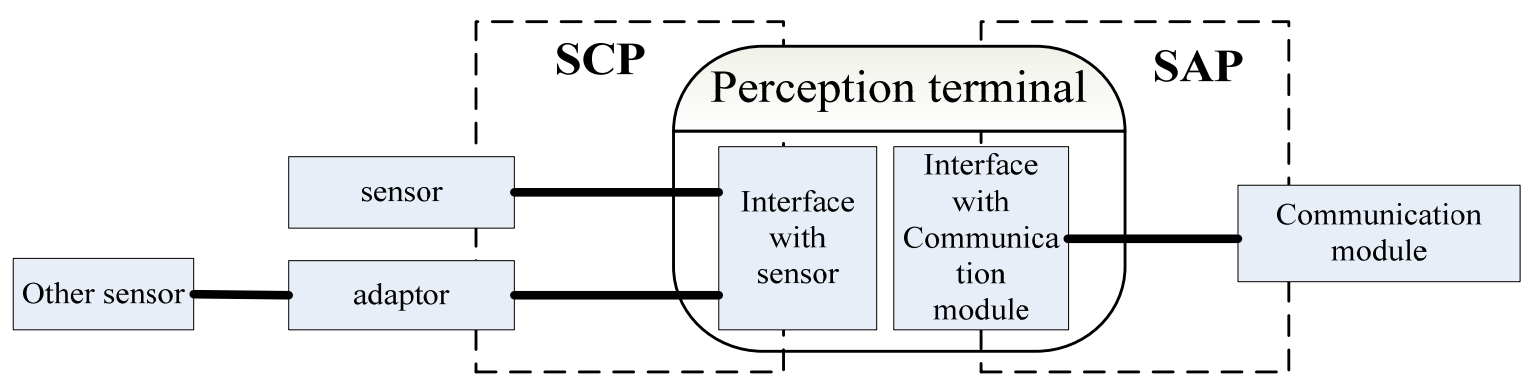

Figure 2. Communication protocols of complex logistics system

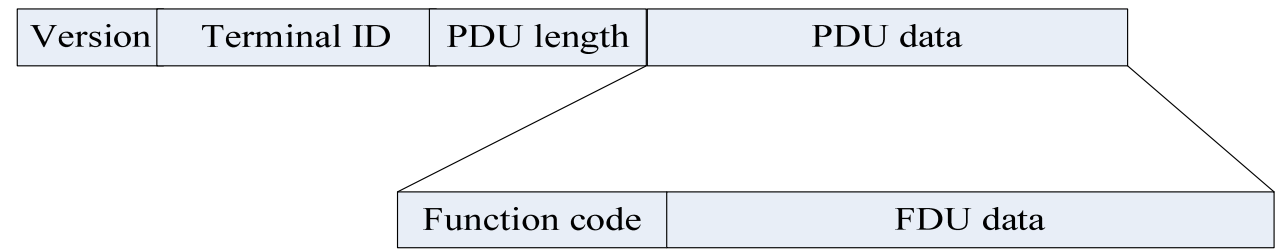

Figure 3.SAP Frame Structure

Table 2. Description of SAP Frame Structure

\begin{tabular}{|c|c|c|c|c|}
\hline Field & Length(byte) & Range & \multicolumn{2}{|c|}{ Explanation } \\
\hline Version & 4 & $\begin{array}{l}\text { X055, } \\
\text { Oxfd, } \\
\text { OXCE, } \\
\text { OXEF }\end{array}$ & \multicolumn{2}{|c|}{ 0x represents hexadecimal } \\
\hline Terminal ID & 16 & Terminal address & \multicolumn{2}{|c|}{ the same as mobile phone IMEI } \\
\hline PDU length & 4 & $0 \sim 4294967295$ & \multicolumn{2}{|l|}{ Variables length } \\
\hline PDU data & Variable & Variable & \multicolumn{2}{|l|}{ Cannot be 0} \\
\hline \multirow[t]{5}{*}{ Function code } & \multirow[t]{5}{*}{2} & \multirow[t]{5}{*}{ Defined } & Function Code & Function \\
\hline & & & F001 F0FF & Sensor data \\
\hline & & & FA01 FAFF & Connection control \\
\hline & & & FB01 FBFF & Data collection rules \\
\hline & & & FC01 FCFF & Data report rules \\
\hline FDU data & Variable & $0 \sim 4294967295$ & \multicolumn{2}{|l|}{ Data unit } \\
\hline
\end{tabular}




\subsubsection{Summery of SCP}

The communication between the perception terminal and sensors is realized by the protocol (see Figure 4). The protocol location in the system is shown in Figure 2.

Therefore, information can be transmitted by the above depicted frame structure, see Figure 4. The meanings and provisions of the frame structure are shown in Table 3.

\subsection{Neural network-based complex logistics system learning and feeding back}

BP (Back Propagation) neural network is introduced, which belongs to feed forward network. The BP neural network algorithm is formed on the basis of gradient descent and its learning process (training) is comprised of the forward propagation and backward propagation. For more details see Figure 5.

\begin{tabular}{|l|l|l|l|l|l|l|}
\hline Lader code & Version & Sensor address & PDU length & The first test & PDU data & The second test \\
\hline
\end{tabular}

Figure 4. SCP Frame Structure

Table 3. Description of SCP Frame Structure

\begin{tabular}{|c|c|c|c|c|}
\hline Field & Length(byte) & Range & \multicolumn{2}{|c|}{ Explanation } \\
\hline Lader code & 2 & $0 \times 55 \mathrm{AA}$ & \multicolumn{2}{|c|}{ 0x represents hexadecimal } \\
\hline Version & 1 & Variable & \multicolumn{2}{|c|}{$\begin{array}{l}1 \text { represents } 1.0, \\
2 \text { represents } 1.1 \\
10 \text { represents } 1.9 \\
11 \text { represents } 2.0 \\
12 \text { represents } 2.1 \\
\ldots\end{array}$} \\
\hline Sensor address & 6 & Sensor ID & & \\
\hline PDU length & 2 & $0 \sim 65535$ & \multicolumn{2}{|l|}{ Variables length } \\
\hline The first test & 2 & & & \\
\hline PDU data & Variable & Variable & \multicolumn{2}{|c|}{ It does not exist when PDU length is 0} \\
\hline The second test & 2 & & \multicolumn{2}{|c|}{ It does not exist when PDU length is 0} \\
\hline \multirow[t]{3}{*}{ Function code } & \multirow[t]{3}{*}{2} & \multirow[t]{3}{*}{ Defined } & Function Code & Function \\
\hline & & & F101 F1FF & Automatic identification \\
\hline & & & F201 F2FF & Data collection \\
\hline FDU data & Variable & $0 \sim 65533$ & Data unit & \\
\hline
\end{tabular}

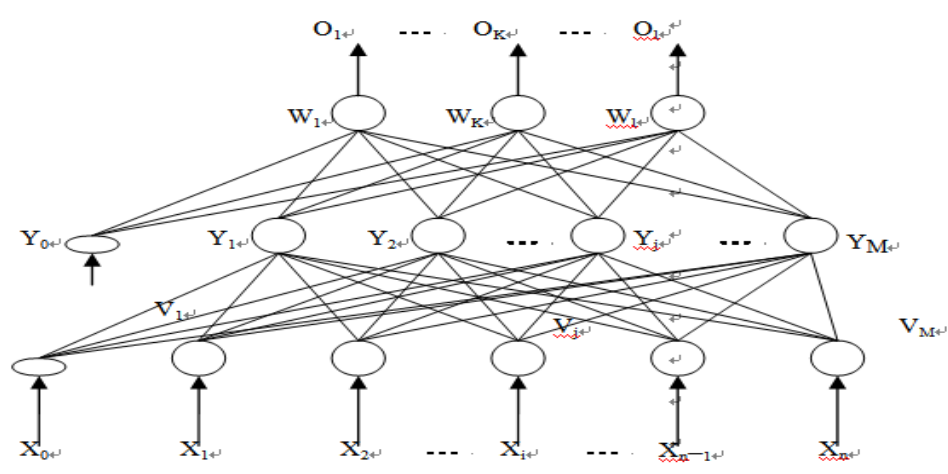

Figure 5. Neural Network Model 
In figure 5, the logistics resources information input vector is:

$X=\left(x_{1}, x_{2}, \cdots, x_{i}, \cdots, x_{n}\right)^{T}$.

The actual logistics resources integration assessment output vector is:

$O=\left(o_{1}, o_{2}, \cdots, o_{k}, \cdots, o_{l}\right)^{T}$.

The desired logistics resources integration assessment output vector is:

$D=\left(d_{1}, d_{2}, \cdots, d_{k}, \cdots, d_{1}\right)^{T}$.

In addition,

$Y=\left(y_{1}, y_{2}, \cdots, y_{j}, \cdots, y_{m}\right)^{T}$

is the vector of the hidden layer.

$V=\left(v_{1}, v_{2}, \cdots, v_{j}, \cdots, v_{m}\right)^{T}$

and

$W=\left(w_{1}, w_{2}, \cdots, w_{k}, \cdots, w_{l}\right)^{T}$

are the connection weights vector between the different layers. The mathematical model is therefore as follows:

For the output layer:

$o_{k}=f\left(\right.$ net $\left._{k}\right) \quad \mathrm{k}=1,2, \cdots, l$

net $_{k}=\sum_{j=0}^{m} w_{j k} y_{j} \quad \mathrm{k}=1,2, \cdots, l$

For the hidden layer

$y_{j}=f\left(\right.$ net $\left._{j}\right) \mathrm{j}=1,2, \cdots, m$

net $_{j}=\sum_{i=0}^{n} w_{i j} x_{i} \quad \mathrm{j}=1,2, \cdots, m$

When the output vector of logistics resources integration assessment is not the desired, the output vector error $E$ is defined as follows:

$E=\frac{1}{2}(d-O)^{2}=\frac{1}{2} \sum_{k=1}^{l}\left(d_{k}-o_{k}\right)^{2}$

Unfold the expression in hidden layer, which is:

$$
\begin{aligned}
E & =\frac{1}{2} \sum_{k=1}^{l}\left[d_{k}-f\left(\text { net }_{k}\right)\right]^{2} \\
& =\frac{1}{2} \sum_{k=1}^{l}\left[d_{k}-f\left(\sum_{j=0}^{m} w_{j k} y_{j}\right)\right]^{2}
\end{aligned}
$$

Unfold the expression in input layer, which is:

$$
\begin{aligned}
E & =\frac{1}{2} \sum_{k=1}^{l}\left\{d_{k}-f\left[\left(\sum_{j=0}^{m} w_{j k} f\left(\text { net }_{j}\right)\right]\right\}^{2}\right. \\
& =\frac{1}{2} \sum_{k=1}^{l}\left\{d_{k}-f\left[\left(\sum_{j=0}^{m} w_{j k} f\left(\sum_{i=0}^{n} v_{i j} x_{i}\right)\right]\right\}^{2}\right.
\end{aligned}
$$

We can see that $E=F\left(w_{j k}, v_{i j}\right)$ and $E$ can be decreased by the change of connection weights. When we input new information to the model, the assessment can be calculated on the premise that $E$ is desired.

The network will be unable to achieve the desired results with a standard BP algorithm as demonstrated by many cases (Vanooyen, A., et al., 1992; Kitano, H., 1990; Martin, T., 1996; Haykin, S., 2008). This paper adopts the following measures to overcome it:

\subsubsection{Increasing momentum term}

The standard BP algorithm only adjusts the weights according to the error gradient direction at time $\mathrm{t}$, without considering the error gradient direction of the previous. This often makes the convergence process slow. In order to make the convergence process faster, a momentum term is introduced in the weight adjustment formula. A weight matrix is represented with $W$, so the weight adjustment formula with the momentum term is:

$\Delta W(t)=\eta \delta X+\alpha \Delta W(t-1)$

Where $\eta$ is the step length, $\delta$ is the error signal, $\alpha$ is the momentum coefficient, and $\alpha \in(0,1)$.

It can be seen that the part of weights of the previous are stacked to the weights of the present and this is the damping for the adjustment in time $t$. It can reduce the oscillation, and increase the training speed when the error surface surges.

\subsubsection{The adjustment of learning rate}

The basic ideology of adaptive learning rate adjustment is to increase the $\eta$ value when error changes greatly, in order to accelerate the network learning rate and to decrease the $\eta$ value when error changes little, in order to rein in the network learning rate. The model is as follows: 


$$
\eta(k+1)= \begin{cases}1.05 \eta(k) & E_{\text {RME }}(k+1)<E_{R M E}(k) \\ 0.7 \eta(k) & E_{R M E}(k+1)>1.04 E_{R M E}(k)(9) \\ \eta(k) & \text { others }\end{cases}
$$

Where $k$ is the training times, $E_{R M E}$ is RMS error $\left(E_{R M E}=\sqrt{\frac{1}{P} \sum_{p=1}^{P}\left(E_{\Sigma}\right)^{2}}\right.$, and where $P$ is the sample).

In the weights adjustment process, the model can ensure step change within a reasonable range and obtain a local minimum or a global minimum.

For example, take a cold chain logistics resources integration. The problems and needs for cold chain business include: interruption of cold chain, real-time temperature monitoring is not implemented, employee theft is severe, cargo transferring process is not standardized, etc. Therefore the key indicators of the cold chain resources integration research includes: the temperature, the humidity, the video interruption period, the temperature interruption period, the combustible gas pressure, the weighbridge measurement difference (employees weight the difference between entering and leaving the treasury), the oil pressure of the vehicle refrigerator, the realtime accessibility of vehicle tracking, and the product information.

Based on the theories above, a simulation experiment (Sensor properties setting, Data collection and management, Network learning, and Feeding back) is carried out. The research shows that the Holographic-based model can realize the data acquisition, learning, feeding back and intelligent analysis for a variety of resources information (including uncertainties). Firstly, the real-time data of the perception layer are transmitted to the data platform through SAP and SCP. For example, for function code F101, its hexadecimal ASCII is: 55 aa $0103010202040500021 \mathrm{c} 85 \mathrm{fl} 01$ e0 85, function code F202, its hexadecimal ASCII is: 55 aa $01030102020405000 \mathrm{a}$ da $84 \mathrm{f} 202000501002000$ 2a 80 bf 8c. The data platform will receive the data: $55 \mathrm{fd}$ ce ef 0123456789 a b c def 000 ae f0 $01 \mathrm{f} 2$ 0255 aa $01030102020405000 a$ da 84 f2 02000501002000 2a 80 bf 8c. Secondly, based on the improved BP algorithms, the system training is finished and the system assessment will be calculated too (see Table 4).
In Table 4, it can be seen that the simulation results are generally accurate except for 'sample8'. More indicators require more corresponding samples. In the case of fewer samples paired with more indicators, the simulation results are still satisfactory. Therefore, the model is credible overall. Ultimately, the applications of the model are shown in Figure 6-9. In Figure 6-9, users can verify the real-time status of logistics resources integration resorting to client system and take actions based on the feedback promptly. All of these ensure the logistics system robustness.

Table 4. Simulation Results

\begin{tabular}{|l|c|c|}
\hline \multirow{2}{*}{ Samples } & \multicolumn{2}{|c|}{ Results } \\
\cline { 2 - 3 } & Actuality & Forecast \\
\hline Sample 1 & 1 & 1 \\
\hline Sample 2 & 1 & 1 \\
\hline Sample 3 & 1 & 1 \\
\hline Sample 4 & 1 & 1 \\
\hline Sample 5 & 1 & 1 \\
\hline Sample 6 & 0 & 0 \\
\hline Sample 7 & 0 & 0 \\
\hline Sample 8 & 0 & 1 \\
\hline Sample 9 & 0 & 0 \\
\hline Sample10 & 0 & 0 \\
\hline
\end{tabular}

\section{Conclusions}

Information technologies have changed modern logistics industries dramatically. With the dissemination of modern technologies, logistics industries have grown much more complex. With resemblance to the organic ecological systems, the aim of logistics resources integration is to ensure the logistics organic ecological system's coordinated development. Furthermore, building integrated and intelligent control systems in an industrial milieu is, obviously, the ultimate target of many professionals in the IT fields, including control, computer, and communication engineers (Filip F.G, 1993).

In this study, we first focus on the 'sensory function' and the 'nerve system'. By investigating the 'sensory function' of logistics systems based on SAP/SCP communication protocol (IoT), we standardized the data with the perceptual subsystem, which in return provides the bases for network learning and feeding back. In addition, this study focuses on the 'nerve system' (Neural Network). This study develops a set of neural network learning and training system by utilizing the real-time sensor data based on the improved algorithm. At last, the 


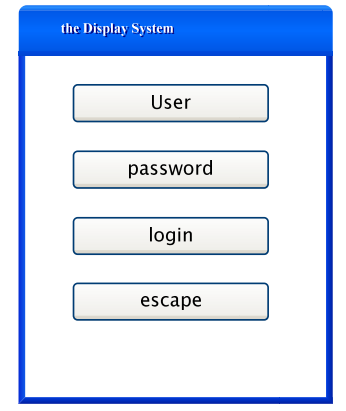

Figure 6. The Display System

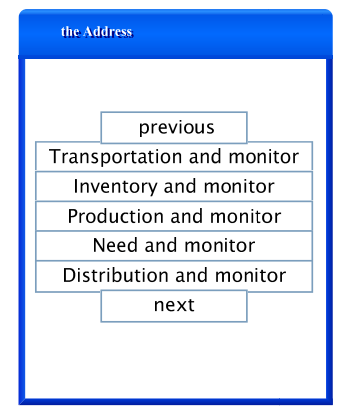

Figure 8. The Address

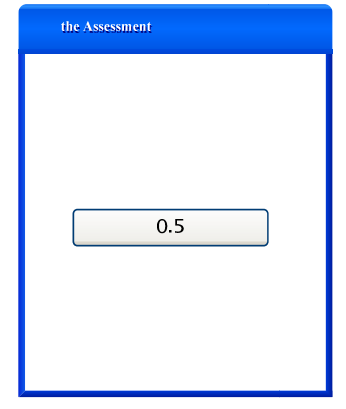

Figure 7. The Assessment

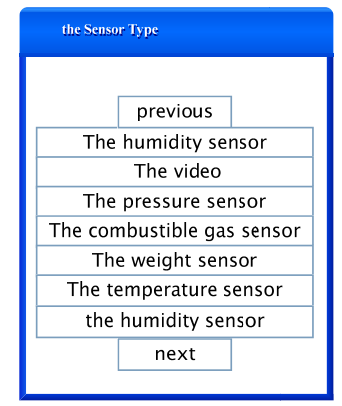

Figure 9. The Sensor Type robust logistics system is developed to realize intelligent analysis in real world environments.

However, this paper has certain limitations and deficiencies. To guarantee the robustness of logistics resources integration, it requires a lot of raw data for simulation. However, IoT technologies are still in the initial stage regarding logistics resources integration. Therefore, the simulation lacks original data. Consequently, the simulation studies are carried out based on laboratory data instead of raw data.

\section{Acknowledgements}

This study has been supported by the National Natural Science Foundation (71132008) and the Fundamental Research Funds for the Central Universities (B12JB00280, B13JB00240), we very much appreciate the support.

\section{REFERENCES}

1. KLIBI, W., A. MARTEL, A. GUITOUNI, The Design of Robust Value-Creating Supply Chain Networks: A Critical Review, Eur. J. of Op. Res., vol. 203(2), 2010, pp. 283-293.

2. MANUJ, I., J. MENTZER, Global Supply Chain Risk Management, J. of Bus. Log., vol. 29(1), 2008, pp. 133-155.
3. LEMPERT, R., D. GROVES, S. POPPER, S. BANKES, A General, Analytic Method for Generating Robust Strategies and Narrative Scenarios, Man. Sc., vol. 52(4), 2006, pp. 514-528.

4. SNYDER, L., Facility Location under Uncertainty: A Review, IIE Trans., vol. 38(7), 2006, pp. 537-554.

5. TOMLIN, B., On the Value of Mitigation and Contingency Strategies for Managing Supply Chain Disruption Risks, Man. Sc., vol. 52(5), 2006, pp. 639-657.

6. SHEFFI, Y., The Resilient Enterprise: Overcoming Vulnerability for Competitive Advantage, MIT Press Books, 2005.

7. LEE, H., Creating Value through Supply Chain Integration, http://www.highbeam.com/doc/1G164972562.html, 2000.

8. LEPAK, D., K. SMITH, M. TAYLOR, Value Creation and Value Capture: $A$ Multilevel Perspective, Ac. Man. Rev., vol. 32(1), 2007, pp. 180-194.

9. BRINTRUP, A., Behaviour Adaptation in The Multi-agent, Multi-objective and Multi-role Supply Chain, Computers in Industry, vol. 61(7), 2010, pp. 636-645. 
10. AKANLE, O., D. ZHANG, Agent-based Model for Optimising Supply-Chain Configurations, Intl. J. of Prod. Ec., vol. 115(2), 2008, pp. 444-460.

11. IAO, J., X. YOU, A. KUMAR, An Agentbased Framework for Collaborative Negotiation in The Global Manufacturing Supply Chain Network, Rob. \& Comp.-Int. Manuf., vol. 22(3), 2006, pp. 239-255.

12. PLOOG, M., T. SPENGLER, Integrated Planning of Electronic Scrap Disassembly and Bulk Recycling, IEEE Intl. Sym. on El. and Environ., 2002.

13. TSERNG, H., S. YIN, S. LI, Developing A Resource Supply Chain Planning System for Construction Projects, J. Constr. Eng. \& Man., vol. 132(4), 2006, pp. 393-407.

14. BHATTACHARYYA, K., Value Sourcing in Supply Chains, Unpublished $\mathrm{PhD}$ diss., Kent State University, Kent, 2011.

15. DYSON, B., N. CHANG, Forecasting Municipal Solid Waste Generation in A Fast-Growing Urban Region with System Dynamics Modelling, Waste Man., vol. 25(7), 2005, pp. 669-679.

16. ZANJANI, A., M. SAEEDI, B. KIANI, A. VOSOOGH, The Effect of The Waste Separation Policy in Municipal Solid Waste Management Using The System Dynamic Approach, Intl. J. of Envir. Health Eng., vol. 1(1), 2012, pp. 1-5.

17. GEORGIADIS, P., M. BESIOU, Environmental and Economical Sustainability of WEEE Closed-loop Supply Chains with Recycling: A System Dynamics Analysis, The Intl. J. of Adv. Man. Tech., vol. 47(5-8), 2010, pp. 475-493.

18. SCHIERITZ, N., A. Größler, Emergent Structures in Supply Chains-A Study Integrating Agent-Based and System Dynamics Modelling, Proc. 36th Ann. Hawaii Intl. Conf. on Sys. Sc., 2003.

19. SODHI, M., B. REIMER, Models for Recycling Electronics End-of-life Products, OR Spectrum, vol. 23(1), 2001, pp. 97-115.

20. PRAJOGO, D., J. OLHAGER, Supply Chain Integration and Performance: The Effects of Long-term Relationships, Information Technology and Sharing, and Logistics Integration, Intl. J. of Prod. Ec., vol. 135(1), 2012, pp. 514-522.

21. BARTON, R., A. THOMAS, Implementation of Intelligent Systems, Enabling Integration of SMEs to HighValue Supply Chain Networks, Eng. App. of AI, vol. 22(6), 2009, pp. 929-938.

22. WAMBA, S., A. CHATFIELD, A Contingency Model for Creating Value from RFID Supply Chain Network Projects in Logistics and Manufacturing Environments, Eur. J. of Inf. Sys., vol. 18(6), 2009, pp. 615-636.

23. HANDFIELD, R., E. NICHOLS, Future Challenges in Supply Chain Management, Introduction to Supply Chain Management, Englewood Cliffs, New Jersey: Prentice-hall, 1999.

24. SAHAY, B., Supply Chain Collaboration: The Key to Value Creation, Work Study, vol. 52(2), 2003, pp. 76-83.

25. NAGARAJAN, M., G. SOŠIĆ, G., GameTheoretic Analysis of Cooperation Among Supply Chain Agents: Review and Extensions, Eur. J. of Op. Res., vol. 187(3), 2008, pp. 719-745.

26. DZITAC, I., B. E. BARBAT, Artificial Intelligence + Distributed Systems $=$ Agents, Intl. J. of Comp., Comm. \& Ctrl, vol. 4(1), 2009 pp. 17-26.

27. VANOOYEN, A., B. NIENHUIS, Improving The Convergence of The Back-propagation Algorithm, Neural Networks, vol. 53(3), 1992, pp. 465-471.

28. KITANO, H., Designing Neural Networks Using Genetic Algorithms with Graph Generation System, Complex Sys., no. 4, 1990, pp. 461-476.

29. MARTIN, T., B. HOWARD, H. MARK, Neural Network Design, Pws Pub, 1996.

30. HAYKIN, S., Neural Networks and Learning Machines, Pearson Academic, 2008.

31. FILIP, F. G, Trends and Tools in Systems Analysis, Modelling, Simulation and Control, Systems, Man and Cyb., Proc. Intl. Conf. 'Syst. Eng. in the Service of Humans', vol. 4, 1993, pp. 413-416. 